\title{
Studio geofisico della colata leucititica a Civita Castellana $(*)$
}

\author{
G. Maruffi $(* *)$ \\ Ricevuto il 16 Gennaio 1967
}

\begin{abstract}
Rrassunto. - Nel quadro dell'attività dell'Istituto di Geofisica Mineraria della Facoltà di Ingegneria dell'Universita di Roma, si è effettuato uno studio geofisico, mediante il metodo geoelettrico, delle lave leucititiche di Civita Castellana-Borghetto e di Fabrica di Roma, tendente a rilevare so tra i due affioramenti citati esista o meno un collegamento.

Ja ricerca ha fornito elementi a farore della seconda ipotesi ed ha permesso di individuare il luogo di fuoriuseita della colata di Civita CastellanaBorghetto.

In appendice sono riportati i risultati di una prospezione radiometrica, effettuata nella stessa zona, diretta a stabilire l'efficacia del metodo per lindividuazione dei contatti, in superficie, tra due formazioni vulcaniche del tipo di quelle affioranti, cioc̀ tra tufi e lava.
\end{abstract}

Sumanx, - $\Lambda$ geo-electrical survey of the lencitic lavas of Civita Castellana-Borghetto and Fabrica di Roma has been carried out at the Institute of Hining Geophysies of the Faculty Engineering of the Rome Unirersity. Purpose of the survey was to determinate en eventual connection between the two outerops.

It is reasonable to conclude that the connection does not exists. The place of exit of the lava of Civita Castellana-Borghetto has been localized.

The results of a radiometric survey carried out in the same area are also enclosed. Purpose of this survey was to see if it is possible to distinguish at the surface the tuli and the lava.

(*) Il presente lavoro fa parte di un programma di studi e di prospezioni geofisiche collegati con ricerche geologiche e condotti sotto gli auspici del C.N.R. con il contributo finanziario del Comitato Nazionale per le scienze Geolngiche e Minerarie. di Roma.

(**) Istituto di Geofisica Mineraria, Facolti di Ingegneria dell'Universit ì 


\section{Prginesse.}

J'apparato vulcanico di Vico è stato recentennente oggetto di un interessante studio geologrico da parte dell'Istituto di Geologia Applicata della Faroltì di Ingegneria dell' Cniversitì di Roma, diretto dal Prof. U. Ventrigrlia.

Jo studio ha permesso di individuare due afforamenti di lava lencititica, l'uno in prossinniti di Civita Castellana e di Borghetto, l'altro a Fabrica di Roma (Fig. 1). Tali masse laviche, che risultano separate in superficie da una vasta coltre di tufi, somo molto sinnili tra loro per le calatteristiche litologiche a petrografiche.

Nel quadro dell'attività dell'Istituto di Geofisica Minemaria della Facolti di Ingegneria di Roma, diretto dal Prof. C. Aquilina, lo scrivente ha effettuato una indagine geofisica nella zoma allo seopo di stabilire se i due affiommenti leucititici menzionati siano in colleganento, al disotto della copertura piroclastica, er appantengano quindi ad mico fenomeno eruttivo o se piuttosto facciano parte di due colate laviche distinte.

La ricerea presenta un certo interesse, oltre che scientifico, anche geoidrologico a geominerario, quest'ultimo in vista di uno sfruttamento industriale della leucite per l'estrazione della potassa.

Lo scrivente desidera ringraziare il Prof. U. Ventriglia per aver inclicato il problema, il suo assistente Dott. P. Mattias per aver messo a disposizione tutti gli elementi del rilievo geologico effettuato. Desidera esprimere la propria gratitudine al Prof. C. Aquilina per aver reso possibile l'efóttuazione della rieera, all'Ing. MI. Bermabini per la costante guida, all'Ing. C. Palozzi per gli utili consigli.

\section{Cenni Geologici.}

La zona oggetto del presente studio è compresa nei fogli 137 e 143 della Carta d'Italia del I.G.MI. e si estende tra gli abitati di Fabrica di Roma, Corchiano, Borgletto e Civita Castellana.

I terreni affioranti nolla zona, di natura prevalentemente vulcanica, sono costituiti da tufi e lava e rappresentano i prodotti di emissione dell'apparato vuleanico di Vico.

Dallo studio gia citato dol Prof. U. Tentrigria e del suo assistente Dott. P. Mattias risulta che le formazioni affiomanti somo rappresentate essernzialmente da ( $\left.{ }^{(}\right)$: 


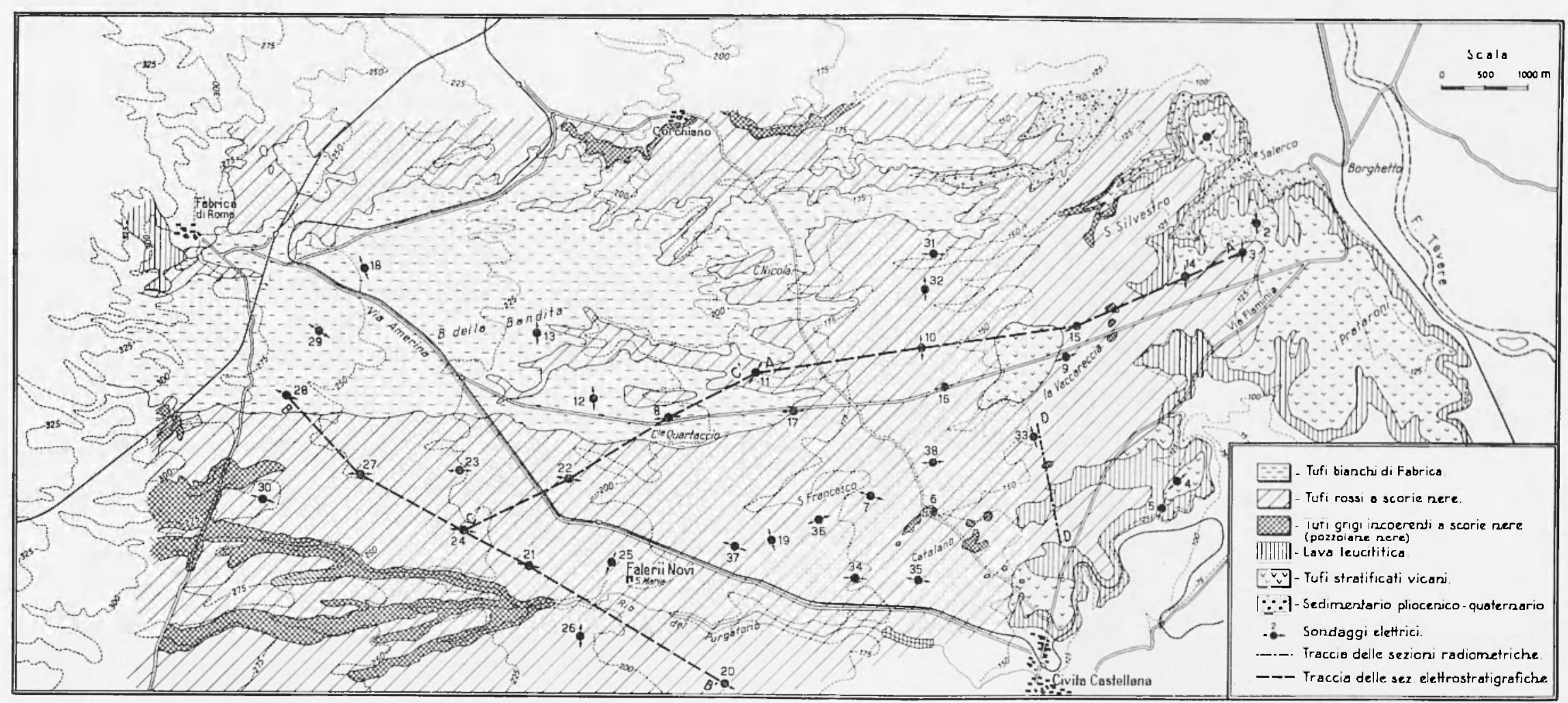

Fig. 1 - Estratto della Carta Geologica (dal rilevamento di P. Mattias). 
- Tufi bianchi di Fabrica: sono tufi litoidi bianeastri, riechi in scorie di colore bianco o giallo; affiorano nella parte Nord-Ovest della Carta Geologica e poggiano sui tufi rossi e scorie nere.

- Tufi rossi a scorie nere: sono tufi leucititici di colore rosso con incluse grosse scorie nere; tale formazione può poggiare sulla lava leucititica ovvero sulle pozzolane nere.

- Tufi grigi incoerenti a scorie nere (pozzolane nere): litologicamente è un materiale scoriaceo, incoerente, a grana minuta e di colore grigio scuro; contiene numerosissime scorie nere in cui si osservano anche cristalli di leucite alterata.

- Lava leucititica: di colore grigio chiaro, ricca in cristalli di leucite del diametro di $1-2 \mathrm{~cm}$. affiora a Civita Castellana e Borghetto ed appare macroscopicamente identica a quella di Fabrica di Roma $\left(^{2}\right)$. Tale formazione poggia sui tufi stratificati vicani ovvero, come nella valle del Tevere e del Salerco, direttamente sulle ghiaie del sedimentario marino; la stessa soggiace ai tufi rossi a scorie nere, talvolta tramite alcuni livelli di pozzolane nere e di tufi stratificati vicani.

- Tufi stratificati vicani: si presentano a grana minuta, incoerenti e di colore marrone, con intercalati livelli di pomici gialle e di lapilli.

- Substrato sedimentario: è costituito da ghiaie, sabbie ed argille di età pliocenica e quaternaria $\left({ }^{*}\right)$.

La ricerca geofisica, di cui la presente nota, è stata effettuata nelle zone di affioramento della lava di Civita Castellana e di Borghetto, dei tufi rossi a scorie nere e dei tufi bianchi di Fabrica di Roma.

\section{SCELTA DEL METODo.}

Una prospezione geofisica su terreni di natura vulcanica si può avvalere dei metodi geoelettrico, magnetometrico e sismico.

Per dare la preferenza ad uno di essi è stata eseguita nella zona in esame un'indagine preliminare sulla possibile utilizzazione dei metodi stessi.

(*) I nomi delle formazioni soprariportati e figuranti anche nella leggenda della Carta Geologica allegata, devono ritenersi provvisori, in quanto la loro esatta definizione, come avvertono i rilevatori, potrà essere fatta soltanto alla fine degli studi attualmente in corso di ultimazione, che si estendono anche alla regione sabazia. 
In particolare, per il metodo magnetometrico, sono state effettuate sulle formazioni presenti alcune misure della componente anomala verticale del campo magnetico terrestre. Tali misure sono state eseguite con la bilancia magnetica di Schmidt secondo due allineamenti pressochè ortogronali. Si è constatato che le anomalie in corrispondenza delle varie formazioni non presentavano tra loro scarti di tale entità da consigliare l'estensione del metodo magnetometrico a tutta la zona da investigare.

Il metodo sismico comporta normalmente l'impiego di esplosivi, impiego che presentava notevoli difficoltà nella zona. Si è voluto tuttavia esaminare se qualche risultato utile potesse ottenersi adottando, quale generatore di vibrazioni, una massa metallica del peso di circa $35 \mathrm{~kg}$. Non si sono ottenuti purtroppo risultati soddisfacenti nelle zone ove il tufo di copertura presentava spessori maggiori di $10-15 \mathrm{~m}$.

Sono stati pertanto scartati il metodo magnetometrico e quello sismico e si ̀̀ adottato il metodo geoclettrico che, d'altra parte, data la natura litologica e petrografica delle formazioni in esame, facera prevedere buoni risultati.

\section{Prospezione geodlettrica.}

Allo scopo di avere una conferma alla presupposta utilizzazione del metodo geoelettrico, sono stati preventivamente effettuati alcuni sondaggi elettrici di taratura sia sulle formazioni affioranti, sia accanto a pozzi e perforazioni meccaniche già esistenti nella zona ed utilizzati per usi irrigui, dei quali si conosceva la stratigrafia. Si è constatato che tra le formazioni in esame ed in particolare tra i tufi e la lava leucititica esisteva una sensibile differenza di resistività tale da consigliare l'applicazione del metodo stesso.

Nelle operazioni di campagna si è adoperata un'apparecchiatura potenziometrica a corrente continua $\left(^{*}\right)$ e si è adottata la disposizione elettrodica di Schlumberger-Sineriz con stese massime tra gli elettrodi di corrente dell'ordine di 500 ed $800 \mathrm{~m}$.

(*) L'apparecchiatura potenziometrica utilizzata permette misure di differenza di potenziale con fondi scala compresi tra $1 \mathrm{mV}$ e $1000 \mathrm{mV}$ e misure di intensità di corrente con fondi scala compresi tra $1 \mathrm{~mA}$ e $3000 \mathrm{~mA}$. Il galvanometro di zero la una sensibilità di $10^{-8}$ Ampere. 
L'indagine è stata impostata inizialmente sull'esecuzione di alcuni sondaggi elettrici verticali distribuiti su allineamenti, che collegavano i diversi affioramenti della lava leucititica; ciò avrebbe doruto permettere di risolvere il tema principale della ricerca, cioè il collegamento tra le due colate di Civita Castellana-Borghetto e di Fabrica di Roma. Successivamente, una volta effettuata una preliminare interpretazione della
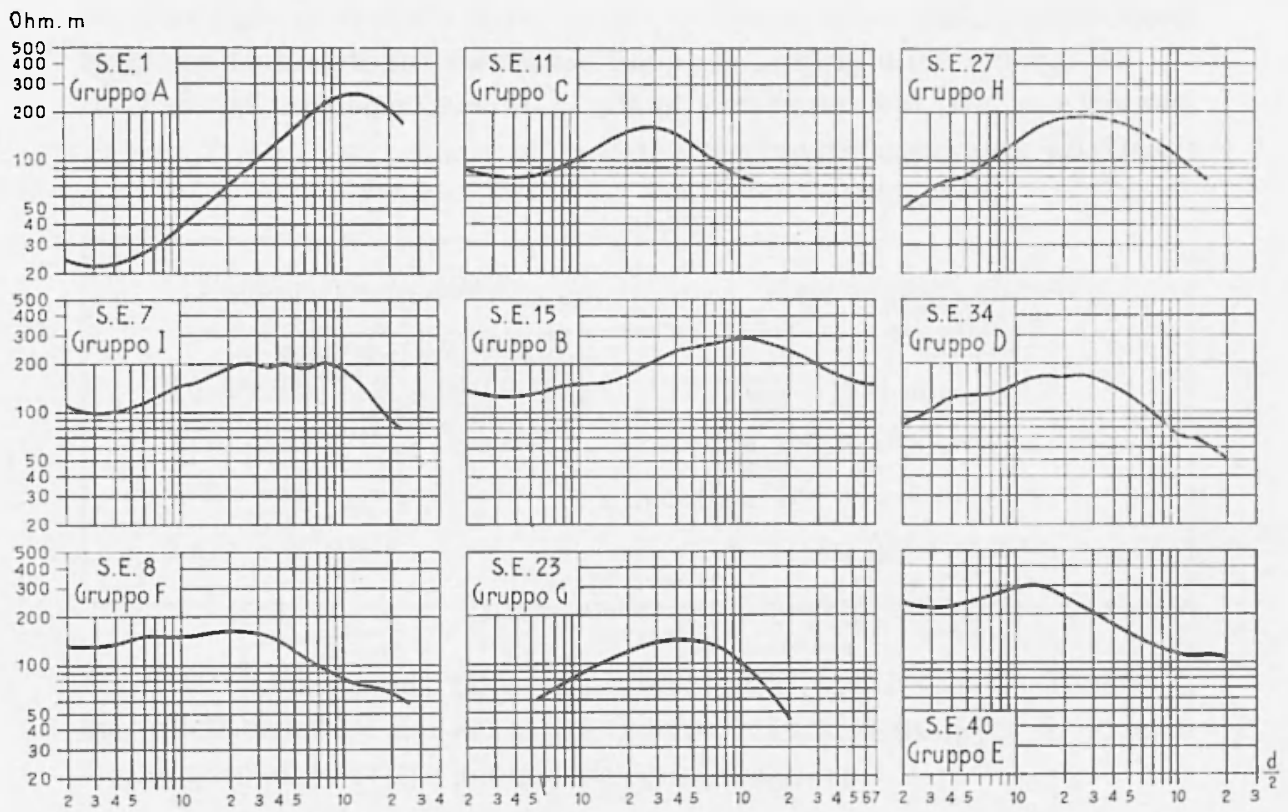

Fig. 2 - Diagrammi delle resistiviti apparenti caratteristiche dei gruppi A (S.E. 1), I (S.E. 7), F (S.E. 8), C (S.E. 11), B (S.E. 15), G (S.E. 23), H (S.E. 27), D (S.E. 34), E (S.E. 40). Le curve 1-8-11 si riferiscono ai sondaggi elettrici di taratura effettuati rispettivamente sugli allioramenti della lava leucititica, del tufo bianco di Fabrica e del tufo rosso a scorie nere.

prima parte del rilievo, è stata eseguita una seconda serie di sondaggi in punti di particolare interesse per la ricostruzione della situazione geologica della zona (Fig. 1).

Per l'interpretazione dei sondaggi elettrici si è ricorsi in primo luogo ad un'interpretazione, sondaggio per sondaggio, mediante il confronto tra le curve di campagna e le curve teoriche. Queste ultime sono state costruite in massima parte utilizzando gli abachi della Compagnie 
Génerale de Geophisique ( $\left.{ }^{4}\right)$ e gli abachi di Cagniard $\left({ }^{3}\right)$; di questi ultimi ne sono stati costruiti altri due per casi di $c_{1} / c_{3}$ diversi rispetto a quelli riportati da Lasfargues ( $\left.{ }^{6}\right)$.

Si è tentata inoltre un'interpretazione globale riunendo in gruppi i sondaggi elettrici che presentavano andamenti simili dei diagrammi delle resistività apparenti. Si sono potute in tal modo effettuare correlazioni tra i risultati forniti dalla interpretazione dei singoli sondaggi elettrici e ricostruire così, in termini di resistività, l'andamento dei vari mezzi individuati.

Di ognuno dei 9 gruppi, nei quali sono stati suddivisi i 41 sondaggi elettrici eseguiti, per semplicità, in Fig. 2, è stata riportata la curva di resistività apparente ritenuta più indicativa.
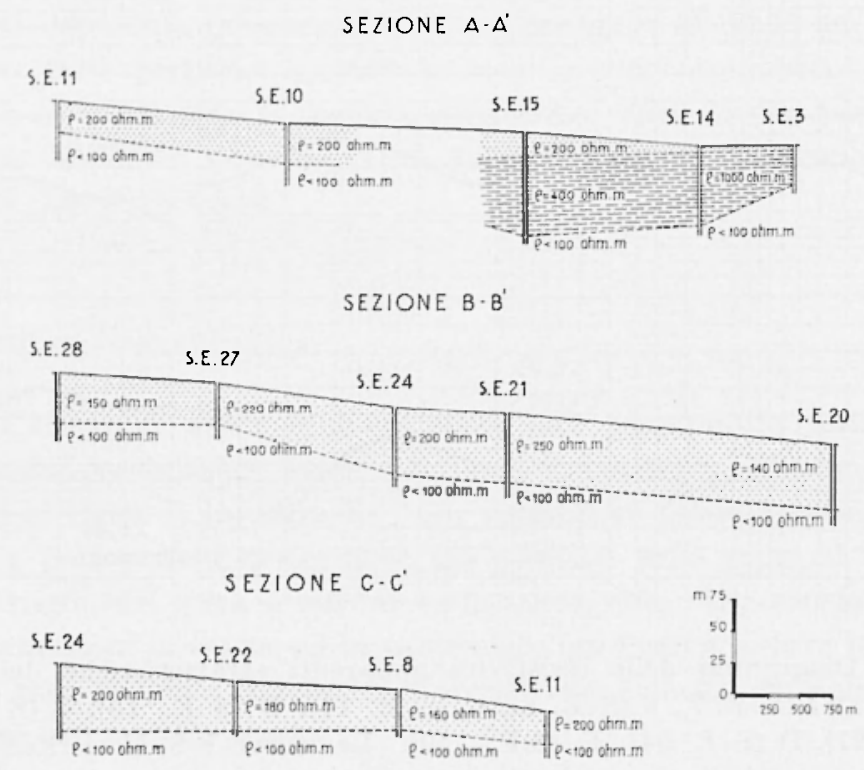

Fig. 3 - Sezioni elettrostratigrafiche.

In particolare, per tre di questi gruppi (A-C-F) sono state sceltecome indicative le curve ottenute con i sondaggi effettuati sugli affioramenti: della lava leucititica (gruppo A, sondaggio n. 1), del tufo rosso a scorie nere (gruppo C, sondaggio n. 11) e del tufo bianco di Fabrica (gruppo F, sondaggio n. 8).

L'interpretazione dei sondaggi ha permesso di costruire le tre sezioni geoelettriche, riportate in Fig. 3 , ritenute le più indicative, fra le: 
varie possibili, per mettere in evidenza l'andamento dei vari mezzi isoresistivi nella zona in esame.

Una volta ottenuta la ricostruzione più probabile dell'andamento dei vari mezzi elettricamente differenti, lo scrivente è passato all'interpretazione, sotto l'aspetto geologico, dei risultati della indagine geoelettrica. Per far ciò sono stati presi in considerazione i risultati dei sondaggi elettrici eseguiti nei punti, ove si conosceva la successione stratigrafica dei terreni e si è potuto quindi attribuire alle varie formazioni geologiche, direttamente interessate dallo studio, i diversi intervalli di valori della resistività indicati in Tabella $\mathrm{I}$.

Tabella I - Valori delle Resistività Relative alle Formazioni in ESAMIE OTIENUTI DAI SONDAGGI ELETTRICI DI TARATURA.

\begin{tabular}{|l|l|}
\hline Formazione in esame & Resistiviti in ohm.m \\
\hline Substrato & Fino a 100 ohm.m \\
Tufo bianco di Fabrica & da 150 a 200 ohm.m \\
Tufo rosso a scorie nere & da 200 a 250 ohm.m \\
Lava leucititica & da 400 a 1000 ohm.m \\
\hline
\end{tabular}

Dal confronto dei valori delle resistività, riportati nella Tabella I, con quelli ottenuti dai sondaggi elettrici effettuati in tutta la zona in esame sono state tratte le seguenti constatazioni:

- Gli alti valori delle resistività, compresi tra 400 e 1000 ohm.m, attribuiti alla lava leucititica, si riscontrano unicamente in tre dei gruppi sopra indicati e precisamente nei gruppi A-B-I.

- I valori medi delle resistività, relativi ai tufi, si riscontrano nei rimanenti gruppi di curve; non è stato però possibile effettuare una distinzione netta tra $\mathrm{i}$ due tipi di tufi in base alle rispettive resistività, in quanto queste presentano valori molto simili tra loro.

- I valori delle resistività inferiori ai 100 ohm.m, attribuiti al substrato, appaiono in tutti i sondaggi, talvolta al di sotto del mezzo a media resistività e talvolta di quello ad alta resistività.

Esaminando la Fig. 1, si nota che tutti i sondaggi elettrici, che hanno individuato mezzi ad alta resistività (sondaggi $\mathrm{n}$. 1-2-3-4-56-7-9-14-15-19), cadono in una zona compresa tra gli abitati di Civita 
Castellana e Borghetto e che si estende verso Est fino alle localitì "San Francesco " e "La Vaccareccia ". In tale zona le quote del tetto del mezzo molto resistivo decrescono, nelle grandi linee, dai sondaggi elettrici n. 6 e n. 7 ai sondaggi n. 4 e n. 5 e da questi ultimi ai sondaggri n. 1, n. 2, n. 3 .

Tutti gli altri sondaggri sono ubicati nella zona compresa tra quelle sopra indicate e l'abitato di Fabrica di Roma. Con tali sondaggi non è stata individuata, per lo meno fino alla profondità di indagine (circa 100 metri), l'esistenza di mezzi molto resistivi e di sufficiente spessore.

I risultati forniti dal rilievo geoelettrico sono stati in seguito correlati con tutti gli elementi geologici inerenti alla zona in esame, ottenuti durante il rilevamento vulcanologico o, successivamente, durante la campagna geofisica. Tali elementi sono risultati sempre in accordo con quanto indicato dall'interpretazione dell'indagine geofisica.

Dalla correlazione dei vari elementi, geofisici e geologici, risulterebbe che la lava affiorante tra Civita Castellana e Borghetto si estende, verso Ovest, al di sotto dei tufi rossi a scorie nere, fino alle località denominate "San Francesco ", "La Vaccareccia " e "San Silvestro ". Nella zona compresa tra quella sopra indicata e Fabrica di Roma non si è riscontrata la presenza di alcun mezzo ad alta resistivita di sufficiente spessore; ciò porterebbe a far escludere la presenza di lava e quindi l'esistenza di una continuità, in profondità, tra i due affiommenti leucititici di Civita Castellana-Borghetto e di Fabrica di Roma, che risulterebbero pertanto appartenere a due colate distinte.

Gli elementi relativi alle quote del tetto della lava di Civita Castellana-Borghetto, forniti dall'interpretazione dei sondaggi elettrici, appartenenti ai gruppi $\Lambda$-B-I, e correlati con i dati geologici acquisiti, mostrano che dette quote sono decisamente più alte nella località denominata "Catalano " e più esattamente nell'area compresa tra i sondaggi n. 6, n. 7, n. 34, n. 36 e l'affioramento di Fosso del Purgatorio, e tendono a decrescere verso Est e Nord-Est. Di conseguenza in tale località appunto potrebbe essere individuata la probabile zona di fuoriuscita della suddetta colata lavica.

\section{Conclusioni.}

L'indagine geofisica effettuata per stabilire se i due affioramenti lencititici di Civita Castellana-Borghetto e di Fabrica di Roma appartengano ad unico fenomeno eruttivo o se piuttosto facciano parte di 
due colate laviche distinte, ha fornito elementi a farore della seconda ipotesi in quanto non ha portato a riconoscere un collegamento tra i due affioramenti menzionati.

Lo stesso studio ha permesso di individuare la presumibile zona di origine della colata di Civita Castellana-Borghetto nella località denominata "Catalano " e precisamente nell'area compresa tra i sondaggi elettrici n. 6, n. 7 , n. 34 , n. 36 .

In definitiva, la ricerca effettuata permette di confermare, ancora una volta, l'efficacia della prospezione geoelettrica che, con impiego non rilevante di personale e di attrezzatura, può risolvere agevolmente e con sufficiente attendibilità problemi del genere di quello proposto.

\section{Prospezione Radiometrica.}

In occasione della ricerca geofisica, di cui si è precedentemente trattato, lo scrivente ha effettuato, nella zona denominata "La Vaccareccia " (Fig. 1), una prospezione radiometrica, mediante misure di radiazioni $\gamma$, allo scopo di stabilire l'efficacia del metodo per l'individuazione dei contatti in superficie tra due formazioni vulcaniche del tipo di quelle affioranti, cioè tra tufo e lava.

Si è utilizzata un'apparecchiatura costituita da un misuratore portatile del tipo $\mathrm{RA}-40$ e da un fotoscintillatore del tipo F-6, entrambi della Italelettronica.

Nelle misure si è usata la costante di tempo massima permessa dall'apparecchio $(10 \mathrm{sec})$ ed in ogni stazione è stato effettuato un elevato numero di letture durante un intervallo di 5 minuti; tali valori sono stati poi mediati.

Misure preliminari hanno chiaramente mostrato che effettivamente tra le formazioni in esame si hanno sensibili differenze nelle intensità di radiazioni, come risulta dalla Tab. II, nella quale sono riportati i valori medi degli impulsi al secondo ottenuti, per i terreni in esame.

Tabella II - VALORI MEd IN IMPULSI AL SECONDO RELATIVI AI TUFI ED alla lava CON UN TUBO Fotoscintillatore tipo F-6.

\begin{tabular}{|l|l|}
\hline \multicolumn{1}{|c|}{ Formazioni in esame } & Impulsi al secondo \\
\hline Tufi rossi a scorie nere & da 140 a 160 \\
Lava leucititica & da 210 a 230 \\
\hline
\end{tabular}


Successivamente sono state eseguite quattro serie di misure lungo un profilo opportunamente scelto, facendo stazione in punti distanti circa 20 metri l'uno dall'altro.

Le quattro serie di misure sono state eseguite rispettivamente:

- con tubo scintillatore tenuto a $50 \mathrm{~cm}$ dal suolo

- con lo stesso tubo, schermato, tenuto a $50 \mathrm{~cm}, 100 \mathrm{~cm} \mathrm{e} 150 \mathrm{~cm}$ dal suolo.

La schermatura dello scintillatore, costituita da un anello di piombo, è stata adottata allo scopo di far giungere all'apparecchiatura soltanto le radiazioni provenienti dal basso secondo un opportuno angolo solido. Si sono cosi potuti ridurre gli effetti massa, topografia e conto di fondo che avrebbero potuto mascherare l'ubicazione dell'eventuale zona di contatto geologico. Le tre serie di misure con tubo scintillatore schermato e tenuto a diverse altezze dal suolo sono state effettuate allo scopo di individuare il sistema che risentisse il meno possibile di eventuali concentrazioni in minerali a più alto tenore di radioattività.

Con l'applicazione dello schermo di piombo le zone di influenza calcolate per le diverse altezze del tubo sono risultate rispettivamente di:

$$
\begin{aligned}
91 \mathrm{~cm}^{2} & \text { per } \mathrm{h}=50 \mathrm{~cm} \\
364 \mathrm{~cm}^{2} & \text { per } \mathrm{h}=100 \mathrm{~cm} \\
822 \mathrm{~cm}^{2} & \text { per } \mathrm{h}=150 \mathrm{~cm} .
\end{aligned}
$$

I risultati delle varie osservazioni, espressi in impulsi al secondo, sono stati riportati integralmente, ciò̀ come direttamente letti all'apparecchio, nei profili di radioattività di Fig. 4.

Dal confronto di detti profili si rilevano i seguenti valori medi della radioattività per raggi $\gamma$ e per tubo schermato:

$$
\begin{aligned}
& 32-33 \mathrm{imp} / \mathrm{sec} \text { per il tufo } \\
& 37-39 \mathrm{imp} / \mathrm{sec} \text { per la lava }
\end{aligned}
$$

valori che di per sè mostrano evidentemente l'applicabilità del metodo in ricerche similari.

In particolare dai profili allegati risulta chiaramente:

- L'efficacia dell'applicazione della schermatura in piombo che ha consentito di ridurre sensibilmente gli effetti perturbatori di maggior rilievo.

- La preferenza da attribuire alle misure effettuate con tubo fotoscintillatore schermato e tenuto alla distanza di $50 \mathrm{~cm}$ dal suolo. 
Il profilo relativo infatti mette in maggior evidenza gli scarti esistenti tra le caratteristiche radioattive delle formazioni in esame e presenta inoltre un andamento più regolare e più chiaramente interpretabile.

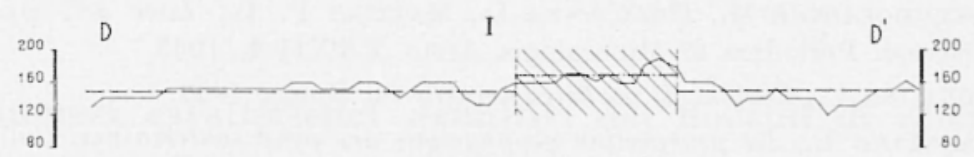

II
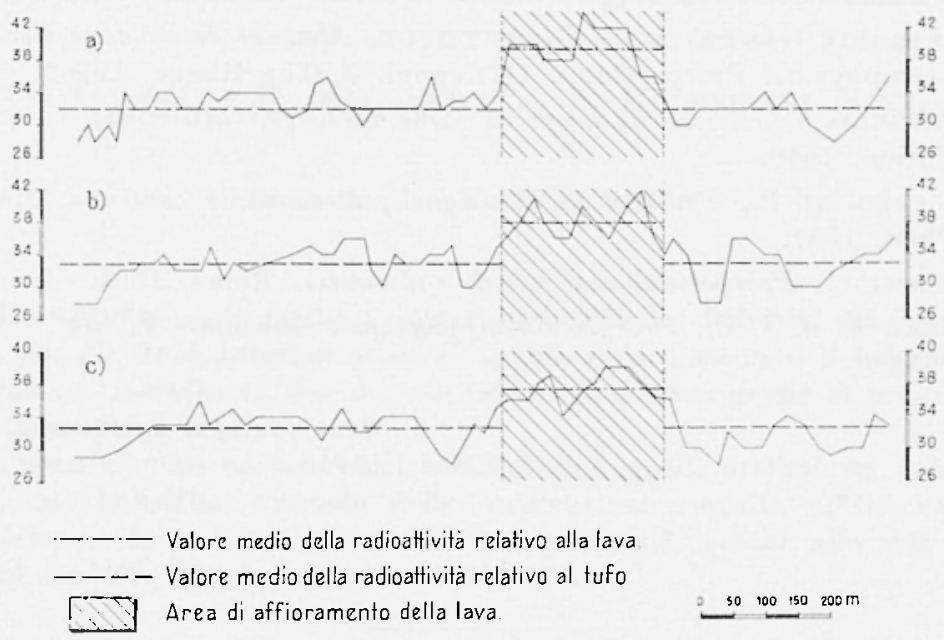

Fig. 4 - Profili radiometrici effettuati con tubo scintillatore non schermato (1); e con tubo scintillatore schermato, tenuto a $50 \mathrm{~cm}$ dal suolo (2a) a $100 \mathrm{~cm}$ dal suolo (2b) e a $150 \mathrm{~cm}$ dal suolo $(2 c)$.

Gli altri tre profili confermano l'andamento generale del fenomeno sebbene con minore chilarezza e regolarità.

\section{Coxclusioni.}

Dilla ricerca effettuata si può dedurre la validità della prospezione radiometrica nell'individuazione dei contatti, in superficie, tra due formazioni a diverso tenore di radioattività, come quelle in esame, cioè tufi e lava lencititica. Risulta inoltre l'efficacia della schermatura di piombo apportata al tubo fotoscintillatore.

La ricerca eflettuata consiglia di approfondire i risultati con un sistema più dettagliato e rigoroso che ei si riserva di applicare in un prossimo futuro. 


\section{BIBLIOGRAFLA}

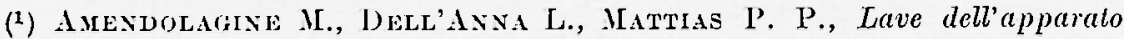
vicano. Periodico di Mineralogia Anno XXXII 1, 1963.

(2) Aquilixa C., Lezioni di geofisiea mineraria. Roma, 1947.

$\left({ }^{3}\right)$ Cagsiard Is, La prospection geopleysique des eaux souterraines. Colloque d'Ankara sur l'hydrologie de la zone aride -UNESCO-Ankara, 1952.

(4) Compagrie Géxína de Geopirsique, Abaques de sondage élechique. "Geophysical Prospecting", III, suppl. 3, The Hagrue, 1955.

$\left({ }^{5}\right)$ Febcieris G., Corso di geofisica mineraria. Levrotto-Bella editori Torino, 1949.

$\left.{ }^{6}\right)$ Lasfargues P'., Prospection électrique par courants continus. MassonParis, 1957 .

(7) P.llozzi C., Prospezioni con melodi ratioallivi. Roma, 1964.

(8) Rotŕ́ E. e. J. P., Prospection geophysique. Gauthiers-Villars - Paris, 1952. 